\title{
Methodological aspects of measuring the resistivity of contacts to high-resistance semiconductors
}

\author{
V.S. Slipokurov', M.M. Dub ${ }^{2}$, A.K. Tkachenko², Ya.Ya. Kudryk ${ }^{1}$ \\ ${ }^{1}$ V. Lashkaryov Institute of Semiconductor Physics, NAS of Ukraine, \\ 41, prospect Nauky, 03650 Kyiv, Ukraine, e-mail: kudryk@isp.kiev.ua \\ ${ }^{2}$ I. Franko Zhitomir State University
}

\begin{abstract}
Proposed has been the method of formation a thermally stable ohmic contact to the diamond without high-temperature annealing with the resistivity $\sim 50$ to $80 \mathrm{Ohm} \cdot \mathrm{cm}^{2}$ when $R_{s}=3 \cdot 10^{7} \mathrm{Ohm} / \square$. Being based on the analysis of correlation dependence between the resistivity of contact and that of semiconductor for the unannealed sample and the sample after rapid thermal annealing it has been shown that variation of the contact resistance on the plate is related with that of semiconductor and may be caused by inhomogeneity of the dopant distribution.
\end{abstract}

Keywords: ohmic contact, diamond, high-resistance semiconductors.

Manuscript received 04.11.14; revised version received 05.04.15; accepted for publication 27.05.15; published online 08.06.15.

In this work, we consider the methodological aspects of measuring the electrophysical parameters of highresistance ohmic contacts to semiconductors, using ohmic contacts to diamond as an example. Natural and artificial diamonds have a number of unique properties such as high thermal conductivity, chemical and radiation resistance, transparency from ultraviolet to radio-frequency range, chemical, radiation resistance and high mobility of the charge carriers. Diamond is a promising material for high power microwave [1-3] and optoelectronic devices, sensors of ionizing radiation, ultraviolet detectors [4]. Developed have already been the Schottky diodes based on diamond with the operating temperature $700{ }^{\circ} \mathrm{C}$, planar transistors with the operating temperature $300^{\circ} \mathrm{C}$, FETs, light-emitting devices [1]. An important element of the semiconductor device is an ohmic contact system, development of which is an essential step of creating technologies of microelectronic devices based on new semiconductor materials.

In this work, we propose to create an ohmic contact to diamond on the basis of $\mathrm{Ti}-\mathrm{Au}$ metallization by using magnetron sputtering on a diamond substrate heated to the temperature $350{ }^{\circ} \mathrm{C}$, which allows to form the contact directly during sputtering. Test specimens were fabricated using bulk polycrystalline $n$-type diamond grown on a silicon substrate. After growing the diamond layer with the thickness of about $100 \mu \mathrm{m}$, silicon was etched. The layered structure of $\mathrm{Ti}(60 \mathrm{~nm})-\mathrm{Au}$ $(100 \mathrm{~nm})$ was deposited using magnetron sputtering on the substrate heated to $350{ }^{\circ} \mathrm{C}$ in a single process cycle. Studied were two types of samples: initial and after rapid thermal annealing (RTA) in vacuum at $800{ }^{\circ} \mathrm{C}$ for $60 \mathrm{~s}$. Using the photolithographic process, the set of test structures to determine the contact resistivity $\left(\rho_{c}\right)$ was created. Type of the test plate and template to determine $\rho_{c}$ is shown in Fig. 1. It was found the current-voltage characteristic measured between these two contacts of template before and after RTA was linear and symmetrical (Fig. 2). For measuring the resistivity of Au-Ti-C contacts, the transmission line method (TLM) was used with linear geometry of contact pads. The essence of this method is as follows: when current flows between two ohmic contacts, the current density is not 
uniformly distributed along the contact length $S$, but decreases exponentially from the edge deep into the contact. For large values of $S$, the current through the contact will not depend on $S$, but it will be determined by the characteristic length of current transfer deep into the contact, the so-called transfer length $\left(L_{t}\right)$. Under this condition the resistance between two rectangular contacts can be written as [5]:

$R_{i}=\frac{R_{s} L_{i}}{W}+2 \frac{L_{t} R_{s}}{W}$,

where $R_{s}$ is the surface resistance of semiconductor between the contacts, $L_{t}=\sqrt{\rho_{c} / R_{s}}, L_{i}$ is the distance between the contacts (see Fig. 1b). After plotting the dependence $R=f\left(L_{i}\right)$, using the slope of the straight line, we calculate $R_{s}$ (Fig. 3a). A line segment intercepted on the $x$-axis by extrapolation to $R=0$ of the dependence $R=f\left(L_{i}\right)$ is equal to $2 L_{t}$, where the contact resistivity $\rho_{c}=L_{t}^{2} R_{s}$.

Both in the sample without annealing and in the annealed one, strong variation of the contact resistivity is observed. In order to identify the reasons for this variation, we will plot the correlation dependences between resistivity of the contact and that of semiconductor for unannealed and processed RTA samples (Fig. 3b). We see that in both cases there is a

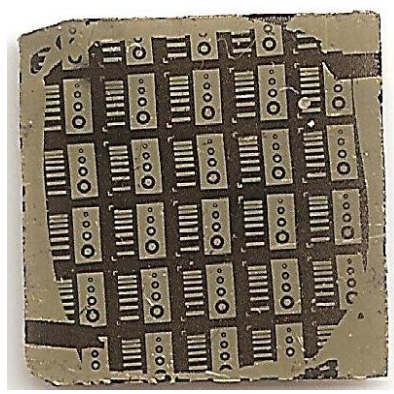

a)

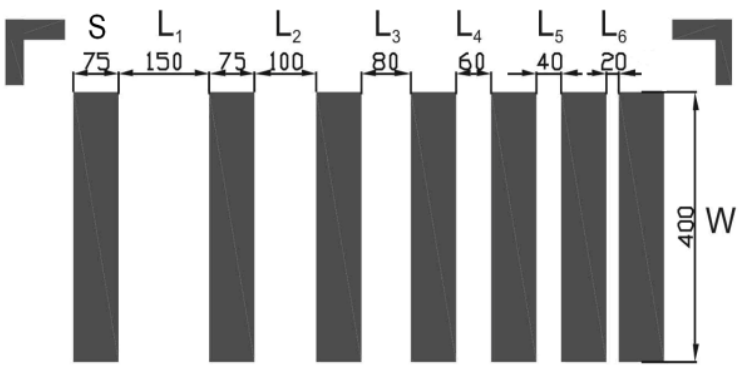

b)

Fig. 1. Test plate (a) and template (b) to determine contact resistivity by using TLM. The sizes are in micrometers.
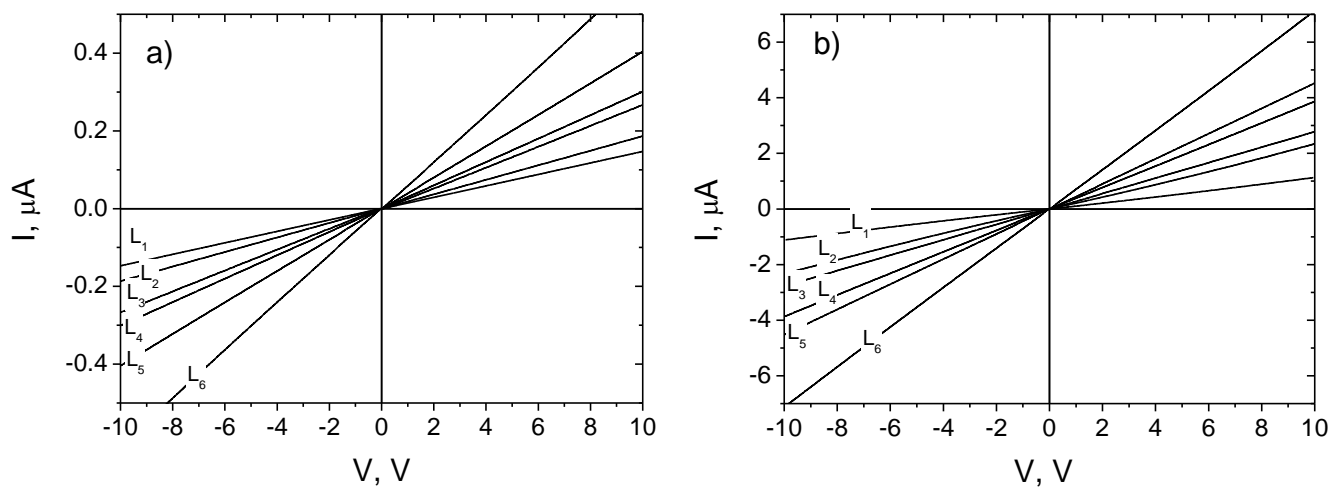

Fig. 2. Current-voltage characteristics of the structure before (a) and after RTA at $800{ }^{\circ} \mathrm{C}$ for $60 \mathrm{~s}(b)$.
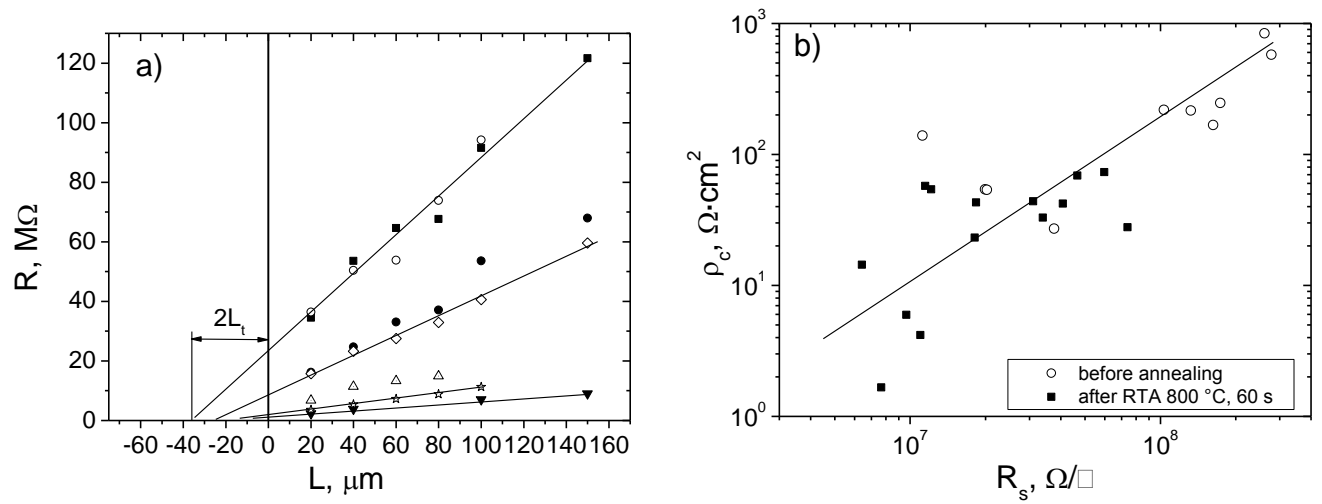

Fig. 3. Determination of the resistivity of contacts and that of semiconductor for the case of Au-Ti-C by using the dependences of the total resistance on a distance between the contacts (a) and correlation dependences of the contact resistance and that of semiconductor before (open marks) and after RTA $800{ }^{\circ} \mathrm{C}$ for $60 \mathrm{~s}$ (filled marks) (b). 
significant positive correlation, which can be explained by a common cause of the change in the resistivity of contact and that of semiconductor - inhomogeneity of doping the diamond substrate.

Fig. $2 b$ shows that for the same values of the substrate resistivity, the semiconductor resistivity is substantially identical before and after annealing, which can be explained by formation of a titanium carbide phase already in the process of magnetron sputtering titanium on the substrate heated to $350^{\circ} \mathrm{C}$. For example, the same was observed in [6], where in the contact Ti-C already at $300{ }^{\circ} \mathrm{C}$ annealing interfacial reactions occured and a transition layer with the thickness $300 \mathrm{~nm}$ was formed. In the work [7], the beginning of the interfacial reactions was fixed at $400{ }^{\circ} \mathrm{C}$.

Thus, we have proposed the method to form ohmic contacts to diamond without high-temperature annealing, which is resistant to rapid thermal annealing at $800{ }^{\circ} \mathrm{C}$ for $60 \mathrm{~s}$. As a result of measuring the contact resistance by using the TLM at room temperature, we has obtained value of $\rho_{c} \sim 50$ to $80 \mathrm{Ohm} \cdot \mathrm{cm}^{2}$ at $R_{s}=3 \cdot 10^{7} \mathrm{Ohm} / \square$.

\section{References}

1. Y. Gurbuz, O. Esame, I. Tekin, W.P. Kang, J.L. Davidson, Diamond semiconductor technology for RF device applications // Solid-State Electronics, 49, p. 1055-1070 (2005).
2. V. Brand, M.E. Saleh, M.P. de Boer, Effects of electrical current and temperature on contamination-induced degradation in ohmic switch contacts // Tribology Intern. 55, p. 8548 (2015).

3. E. Alemanno, M. Martino, A.P. Caricato, M. Corrado, C. Pinto, S. Spagnolo, G. Chiodini, R. Perrino, G. Fiore, Laser induced nano-graphite electrical contacts on synthetic polycrystalline CVD diamond for nuclear radiation detection // Diamond \& Related Materials, 38, p. 32-35 (2013).

4. C.A. Hewett, M.J. Taylor, J.R. Zeidler, and M.W. Geis, Specific contact resistance measurements of ohmic contacts to semiconducting diamond // J. Appl. Phys. 77, p. 755 (1995).

5. H. Morkoc, Handbook of Nitride Semiconductors and Devices, vol. 2. Electronic and Optical Processes in Nitrides. Wiley-VCH, 2008.

6. Y. Zhu, B. Zheng, W. Yao, L. Cao, The interface diffusion and chemical reaction between a Ti layer and a diamond substrate // Diamond \& Related Materials, 8, №6, p. 1073-1078 (1999).

7. Van der Weide, R.J. Nemanich, Interface reactions of titanium on single crystal and thin film diamond analyzed by UV photoemission spectroscopy // Applications of diamond films and related materials; Proc. 1st Intern. Conf., Auburn, AL, Aug. 17-22, 1991, p. 359-364. 